\title{
Exploring collider aspects of a neutrinophilic Higgs doublet model in multilepton channels
}

\author{
Katri Huitu, ${ }^{*}$ Timo J. Kärkkäinen, ${ }^{\dagger}$ and Subhadeep Mondal ${ }^{\ddagger}$ \\ Department of Physics, and Helsinki Institute of Physics, \\ P. O. Box 64, FI-00014 University of Helsinki, Helsinki, Finland \\ Santosh Kumar Rai ${ }^{\S}$ \\ Regional Centre for Accelerator-based Particle Physics, Harish-Chandra Research Institute, \\ HBNI, Jhusi, Allahabad 211019, India
}

(Received 14 December 2017; published 28 February 2018)

\begin{abstract}
We consider a neutrinophilic Higgs scenario where the Standard Model is extended by one additional Higgs doublet and three generations of singlet right-handed Majorana neutrinos. Light neutrino masses are generated through mixing with the heavy neutrinos via the Type-I seesaw mechanism when the neutrinophilic Higgs gets a vacuum expectation value (VEV). The Dirac neutrino Yukawa coupling in this scenario can be sizable compared to those in the canonical Type-I seesaw mechanism owing to the small neutrinophilic Higgs VEV giving rise to interesting phenomenological consequences. We have explored various signal regions likely to provide a hint of such a scenario at the LHC as well as at future $e^{+} e^{-}$colliders. We have also highlighted the consequences of light neutrino mass hierarchies in collider phenomenology that can complement the findings of neutrino oscillation experiments.
\end{abstract}

DOI: 10.1103/PhysRevD.97.035026

\section{INTRODUCTION}

The discovery of the $125 \mathrm{GeV}$ Higgs boson [1,2] has been a remarkable achievement of the Large Hadron Collider (LHC). This has provided us a closure regarding the predictions of the Standard Model (SM). While our quest toward understanding the physics beyond the Standard Model (BSM) continues, the $13 \mathrm{TeV}$ run of the LHC is expected to make a big impact in terms of both higher energy reach and better precision by accumulating a huge amount of data at large luminosity. The enigma of the nonzero neutrino mass has pushed the theorists as well as experimentalists to develop new theories and experimental techniques in order to establish the right theoretical pathway toward unveiling the true nature of neutrino mass generation. The neutrino oscillation experiments have established the fact that at least two of the three light neutrinos are massive, and that they have sizable mixing among themselves (for a review, see [3]). The SM, lacking

\footnotetext{
*katri.huitu@ @elsinki.f

†timo.j.karkkainen@helsinki.f

*subhadeep.mondal@helsinki.fi

§skrai@hri.res.in
}

Published by the American Physical Society under the terms of the Creative Commons Attribution 4.0 International license. Further distribution of this work must maintain attribution to the author(s) and the published article's title, journal citation, and DOI. Funded by SCOAP ${ }^{3}$. any right-handed neutrinos, is unable to account for these phenomena. This has led to a plethora of scenarios leading to neutrino mass generation [4-12]. As the resulting neutrino mass eigenstates may be either Dirac or Majorana type, both scenarios have potentially unique signatures [13-23] in the collider experiments. The LHC Collaborations have put forth significant effort to extract any possible information about such scenarios from the accumulated data, and the null results so far have only been able to constrain the parameter space of various neutrino mass models [24-29].

In the post-Higgs discovery LHC era, the true nature of the scalar sector remains another vital area of interest. The natural question that arises is whether the $125 \mathrm{GeV}$ Higgs is the only scalar as predicted by the SM or other exotic scalars exist alongside, as predicted by various BSM theories including some of the neutrino mass models $[10,30]$. The measurements of couplings of the $125 \mathrm{GeV}$ Higgs with known SM particles have so far been consistent with the SM predictions [31]. Thus, even if this Higgs boson were indeed part of a larger scalar sector, its mixing with the other states would be small. There are still enough uncertainties in these measurements to allow new exotic scalar multiplets. Unless the LHC observes some hint of a new scalar, our only hope lies in the precision measurements of the Higgs couplings in order to constrain the BSM physics scenarios. Meanwhile, there has been a long term interest in the simplest two-Higgs doublet models (2HDM) 
(for a review, see [32]) which are also strongly motivated by supersymmetric scenarios. A two-Higgs doublet model predicts the presence of two $C P$-even, one $C P$-odd and two charged Higgses, one of the $C P$-even Higgs states being the $125 \mathrm{GeV}$ Higgs boson. Despite the presence of these additional scalar states, the mixing between the two doublets can be arranged so that the other scalars are practically decoupled from the SM Higgs. In such cases, the interaction of the SM-like Higgs with the exotic scalars may be so suppressed that any hint of such interactions can be very hard to pick up even with the precision measurements at the LHC. The hope of finding these scalars, therefore, lies in their direct search. While the increasing center-of-mass energy at the LHC can probe heavier exotic particles, extracting any new physics information from the tremendous amount of collected data also faces the increasing challenge of tackling the QCD background. Hence looking for lepton-enriched final states is understandably efficient in suppressing the SM background contributions and probing new physics scenarios which can potentially give rise to lepton-rich final states.

In this work, we consider a 2HDM where the additional Higgs doublet has an odd $\mathcal{Z}_{2}$ symmetry charge opposite to all the SM particles, preventing it from interacting directly with the leptons and quarks. One can additionally incorporate right-handed neutrinos in the model with similar transformation property under $\mathcal{Z}_{2}$ symmetry as the new Higgs doublet. One can thus generate Dirac neutrino mass terms when the $\mathcal{Z}_{2}$ breaks spontaneously and the new Higgs doublet gets a vacuum expectation value (VEV). This class of models, known as neutrinophilic Higgs doublet models $(\nu \mathrm{HDM})$, has been proposed long ago [33-35] and the relevant phenomenology has been studied quite extensively [36-44]. In principle, one can also generate Majorana neutrino mass terms in such a scenario, since a Majorana mass term for the additional right-handed neutrinos does not break the $\mathcal{Z}_{2}$ symmetry but breaks the accidental lepton number symmetry by two units $(\Delta L=2)$. Such a neutrino mass generation mechanism looks very similar to the Type-I seesaw [4-7] case, save for the fact that one uses the neutrinophilic Higgs VEV instead of electroweak VEV in order to generate the light-heavy neutrino mixing. The advantage of having the additional Higgs doublet to generate nonzero neutrino masses is that the additional VEV can be very small ${ }^{1}$ in order to counter the smallness of the light neutrino masses which would otherwise be fit with a very small Dirac neutrino Yukawa coupling that has no significant collider phenomenological aspects.

Depending on whether the nonzero neutrinos are Dirac or Majorana type, the collider signals of a $\nu$ HDM scenario can be very different. When Majorana neutrinos exist, a smoking gun signal would be lepton number violating final

\footnotetext{
${ }^{1}$ This is also preferred from the naturalness argument [45].
}

states. In this work, instead of looking for direct heavy neutrino production, we have considered the production of the neutrinophilic charged Higgs $\left(H^{ \pm}\right)$and explored its various possible decay modes. There are some earlier studies on the charged Higgs in similar scenarios emphasizing its decay into a charged lepton and a heavy neutrino in the process [44]. We show that even cleaner signals can be obtained using this decay mode with higher lepton multiplicity where the SM background is practically nonexistent. We also show that sizable signal event rates can be obtained with other possible decay modes of the $H^{ \pm}$, which can serve as complementary channels in probing a $\nu$ HDM-like scenario. We perform our analysis using the $13 \mathrm{TeV}$ LHC as well as an $e^{+} e^{-}$collider with $1 \mathrm{TeV}$ center-of-mass energy. In the process, one can extract information on the neutrino sector parameters also. We show that a very clean indication of the neutrino mass hierarchy can be obtained from the multiplicity of the charged leptons in the final state even after a rigorous collider simulation. Such information can be very useful in complementing the neutrino oscillation experiments.

\section{MODEL}

In the $\nu$ HDM model, the particle content of the SM is extended by one additional Higgs doublet $\left(\phi_{\nu}\right)$ and three generations of SM gauge singlet right-handed neutrinos $(N)$. A discrete $\mathcal{Z}_{2}$ symmetry is introduced, under which both $\phi_{\nu}$ and $N_{i}, i=1,2,3$, are odd while all the SM fields are even. The most general scalar potential involving the two Higgs doublets is given by

$$
\begin{aligned}
V_{\mathrm{sc}}= & -m_{1}^{2} \phi^{\dagger} \phi+m_{2}^{2} \phi_{\nu}^{\dagger} \phi_{\nu}-m_{3}^{2}\left(\phi^{\dagger} \phi_{\nu}+\phi_{\nu}^{\dagger} \phi\right) \\
& +\frac{\lambda_{1}}{2}\left(\phi^{\dagger} \phi\right)^{2}+\frac{\lambda_{2}}{2}\left(\phi_{\nu}^{\dagger} \phi_{\nu}\right)^{2} \\
& +\lambda_{3}\left(\phi^{\dagger} \phi\right)\left(\phi_{\nu}^{\dagger} \phi_{\nu}\right)+\lambda_{4}\left(\phi^{\dagger} \phi_{\nu}\right)\left(\phi_{\nu}^{\dagger} \phi\right) \\
& +\frac{\lambda_{5}}{2}\left[\left(\phi^{\dagger} \phi_{\nu}\right)^{2}+\left(\phi_{\nu}^{\dagger} \phi\right)^{2}\right],
\end{aligned}
$$

where a nonzero $m_{3}$ explicitly breaks the $\mathcal{Z}_{2}$ symmetry in the model. In the absence of this term, the $\mathcal{Z}_{2}$ symmetry can be broken spontaneously by VEV $v_{\nu}$ of the field $\phi_{\nu}$, while the standard electroweak symmetry is broken when $\phi$ acquires a VEV, $v_{\phi}$.

Let us first discuss a framework, where $m_{3}=0$, i.e. $\mathcal{Z}_{2}$ symmetry is broken only spontaneously in order to generate light neutrino masses and mixing. The model is constrained by sterile neutrino searches, effective number of neutrinos and amount of ${ }^{4} \mathrm{He}$ required in big bang nucleosynthesis $(\mathrm{BBN})$, observed temperature anisotropies of cosmic microwave background (CMB) and astrophysical limits.

Because of an instability of right-handed neutrinos $N_{i}$ induced by their mixing to left-handed neutrinos, the mixing strength between $\nu_{\ell}, \ell=e, \mu, \tau$, and $N_{i}$, that is, 
$\left|U_{\ell i}\right|$, can be probed by sterile neutrino searches. In semileptonic meson decays, $N_{i}$ are produced and can subsequently decay to charged leptons and mesons. Present constraints on $\left|U_{e i}\right|^{2}$ and $\left|U_{\mu i}\right|^{2}$ allow a region where their magnitude is of order $10^{-10}$ to $10^{-6}$, assuming $M_{N_{i}}<2 \mathrm{GeV}$ [29]. For tau-sterile mixing, $\left|U_{\tau i}\right|^{2} \lesssim 10^{-4}$, assuming $M_{N_{i}}<0.3 \mathrm{GeV}$.

In $\nu \mathrm{HDM}$, however, we found the model favoring even lower values of active-sterile mixing, of order $\left|U_{\ell i}\right|^{2} \sim$ $10^{-18}$ to $10^{-12}$, at $M_{N_{i}}=1 \mathrm{GeV}$, and even lower for higher Majorana neutrino masses (see Fig. 1). The largest and smallest active-sterile mixings are driven by $U_{\tau 3}$ and $U_{\tau 1}$ elements. Therefore all the active-sterile mixing elements fall between them: $\left|U_{\tau 1}\right|<\left|U_{\ell i}\right|<\left|U_{\tau 3}\right|$. The matrix elements are proportional to $M_{N}^{-1 / 2}$; therefore $C_{1} \frac{1}{\sqrt{M_{N}}}<$ $\left|U_{\ell i}\right|<C_{2} \frac{1}{\sqrt{M_{N}}}$ with some constants $C_{1}$ and $C_{2}$. They are deduced from Fig. 1 , having values $C_{1}=4.34 \times 10^{-9} \mathrm{MeV}^{1 / 2}$ and $C_{2}=2.34 \times 10^{-6} \mathrm{MeV}^{1 / 2}$. The matrix elements then belong to the following interval:

$$
1.4 \times 10^{-10} \sqrt{\frac{\mathrm{GeV}}{M_{N_{i}}}} \lesssim\left|U_{\ell i}\right| \lesssim 7.4 \times 10^{-8} \sqrt{\frac{\mathrm{GeV}}{M_{N_{i}}}} .
$$

In addition, the constraints for $\left|U_{\ell i}\right|$ were derived from assumption that the branching ratios for $N_{i}$ decay are dominant. This is not applicable for $\nu \mathrm{HDM}$, since then the decay modes of right-handed neutrinos are dominated by decays to invisible particles.

As the model is unconstrained by semileptonic and leptonic decay modes, the lower bound for $M_{N_{i}}$ arises from BBN. In the early universe the right-handed neutrinos must be heavy enough to fall off from the thermal equilibrium before BBN. This is due to the latest results

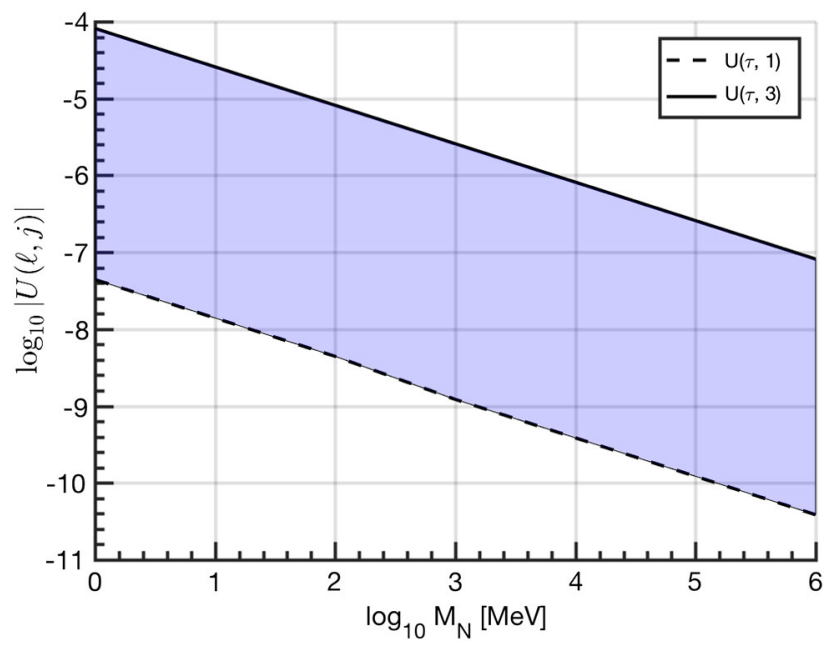

FIG. 1. Absolute values of active-sterile mixing block matrix elements as a function of heavy neutrino mass $M_{N}$. All the activesterile elements fall in the blue band. for the effective number of neutrinos $\left(N_{\nu}=3.15 \pm 0.23\right)$ by PLANCK [46], which forbids large interference from right-handed neutrinos. This leads to a constraint $M_{N_{i}} \gtrsim 100 \mathrm{MeV}$.

In addition neutrinophilic VEV $v_{\nu}$ is constrained from both above and below. Ultralight VEV is forbidden by astrophysical constraints: $v_{\nu} \gtrsim \mathcal{O}(\mathrm{eV})$ [47,48]. On the other hand, the surface energy density associated with the domain wall arising from discrete $\mathcal{Z}_{2}$ symmetry breaking is $\eta \sim v_{\nu}^{3}$ [49]. The effect of these domain walls to the temperature anisotropies of $\mathrm{CMB}$ is

$$
\frac{\Delta T}{T} \approx \frac{G \eta}{H_{0}},
$$

where $G$ is Newton's gravitational constant, $H_{0}$ is the Hubble constant, and we have assumed $\lambda_{i}<1$ [50]. Since the observed temperature anisotropies by PLANCK are $\sim 10^{-5}$, the birth of a domain wall will not contradict cosmological data if the VEV is small. If we require the contribution to $\mathrm{CMB}$ temperature anisotropies not to exceed the experimental limit, together with the astrophysical constraints, we get

$$
\mathcal{O}(\mathrm{eV}) \lesssim v_{\nu} \lesssim \mathcal{O}(\mathrm{MeV})
$$

In order to apply perturbative theory to $\nu \mathrm{HDM}$, the absolute values of the elements of the light neutrino Yukawa coupling matrices must be $\sqrt{4 \pi}$ at most. We performed a global fit to available neutrino oscillation data to calculate the matrix elements, assuming normal neutrino mass ordering, higher $\theta_{23}$ octant, and no $C P$ violation. We found the dependence of the largest Yukawa coupling of $v_{\nu}$ and $M_{N}$ to be

$\max \left|Y\left(v_{\nu}, M_{N}\right)\right| \approx 0.629 \times \frac{100 \mathrm{keV}}{v_{\nu}} \times \sqrt{\frac{M_{N}}{100 \mathrm{GeV}}}$.

The dependence is illustrated in Fig. 2.

The breaking of $\mathcal{Z}_{2}$ symmetry is necessary in order to generate light neutrino masses within the framework of this model by means of their mixing with heavy right-handed neutrinos. One can add the following Yukawa interaction and Majorana neutrino mass terms to the Lagrangian while keeping the $\mathcal{Z}_{2}$ parity unbroken,

$$
\mathcal{L}_{\text {add }}=y_{\nu}^{i j} \bar{L}_{i} \phi_{\nu} N_{j}+\frac{1}{2} m_{R}^{i j} N_{i}^{c} N_{j}+\text { H.c. },
$$

where $m_{R}^{i j}$ represents the Majorana mass terms corresponding to the right-handed neutrinos. Once $\phi_{\nu}$ acquires a VEV, the Yukawa term gives rise to Dirac neutrino mass terms, $m_{D}^{i j}=v_{\nu} \times y_{\nu}^{i j}$.

The physical Higgs sector now consists of two neutral $C P$-even $\left(h, H_{\nu}\right)$, one neutral $C P$-odd $\left(A_{\nu}\right)$, and the charged 


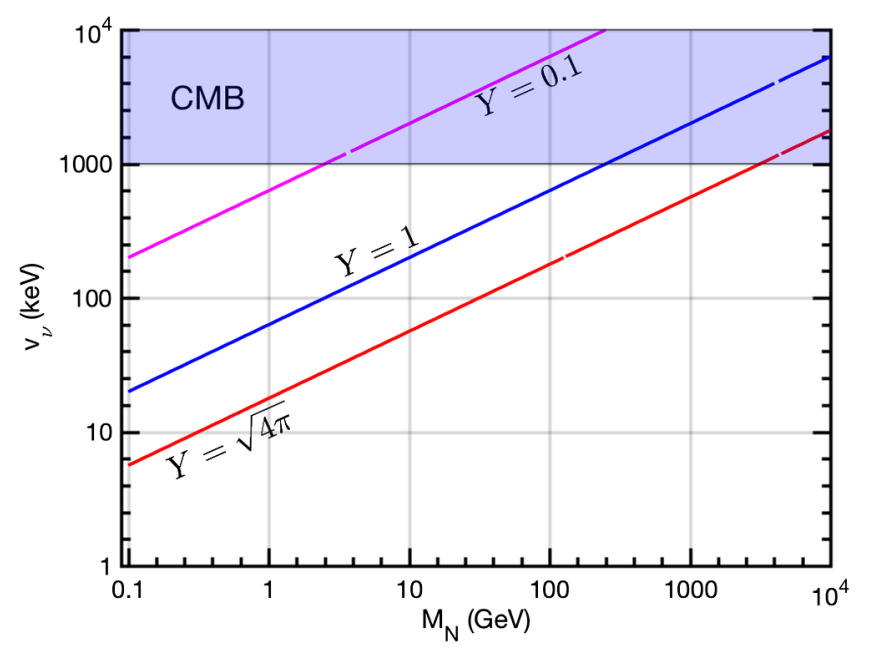

FIG. 2. Yukawa contours on the $\left(M_{N_{i}}, v_{\nu}\right)$ plane. The lines corresponding to the neutrino Yukawa couplings $Y=0.1,1, \sqrt{4 \pi}$ are drawn. Below the red $Y=\sqrt{4 \pi}$ line, the theory is nonperturbative. Blue-shaded region denoted "CMB" is excluded due to restrictions of $\mathrm{CMB}$ temperature anisotropies induced by domain walls. The available parameter space is restricted also from BBN requirement $M_{N_{i}} \gtrsim 0.1 \mathrm{GeV}$.

Higgs $\left(H_{\nu}^{ \pm}\right)^{2}$ In the case when $m_{3}=0$, the physical mass eigenvalues at tree level are given by

$$
\begin{aligned}
m_{h} & =\sqrt{\lambda_{1} v_{\phi}^{2}}, \quad m_{H_{\nu}}=\sqrt{\lambda_{2} v_{\nu}^{2}} \\
m_{A_{\nu}} & =\sqrt{-\lambda_{5} v^{2}}, \quad m_{H_{\nu}^{ \pm}}=\sqrt{-\frac{v^{2}}{2}\left(\lambda_{4}+\lambda_{5}\right)},
\end{aligned}
$$

where $v=\sqrt{v_{\phi}^{2}+v_{\nu}^{2}} \cdot v_{\nu}$ being small, terms proportional to $v_{\nu}^{n}$ (where $\left.n>2\right)$ have been neglected. Note that the mixing angle between the SM and neutrinophilic Higgs states are proportional to the ratio $\frac{v_{\nu}}{v_{\phi}}$ and can be safely neglected since we assume $v_{\nu} \ll v_{\phi}$. Under this circumstance, the $C P$-even neutrinophilic Higgs $\left(H_{\nu}\right)$ is always light and the heavy neutrino almost always decays into $H_{\nu}$ and a light neutrino resulting in an opposite-sign dilepton signal for a charged Higgs pair production channel [40]. However, if the explicit symmetry breaking term is present in the Lagrangian, i.e. $m_{3} \neq 0$, the mass eigenvalues are given by

$$
\begin{aligned}
m_{h} & =\sqrt{\lambda_{1} v_{\phi}^{2}}, \quad m_{H_{\nu}}=\sqrt{m_{3}^{2} \frac{v_{\phi}}{v_{\nu}}+\lambda_{2} v_{\nu}^{2}} \\
m_{A_{\nu}} & =\sqrt{m_{3}^{2} \frac{v_{\phi}}{v_{\nu}}-\lambda_{5} v^{2}}, \quad m_{H_{\nu}^{ \pm}}=\sqrt{m_{3}^{2} \frac{v_{\phi}}{v_{\nu}}-\frac{v^{2}}{2}\left(\lambda_{4}+\lambda_{5}\right) .}
\end{aligned}
$$

\footnotetext{
${ }^{2}$ We have assumed the scalar potential to be $C P$ invariant.
}

Now the neutrinophilic $C P$-even Higgs can be heavy depending on our choice of $m_{3}, m_{H_{\nu}} \simeq \sqrt{m_{3}^{2} \frac{v_{\phi}}{v_{\nu}}}$. A heavy $H_{\nu}$ and (or) $A_{\nu}$ opens up the possibility of a cascade decay via heavy neutrinos resulting in multilepton signals of such a scenario that we intend to explore. In the limit $m_{3} \rightarrow 0$, the symmetry of the theory is enhanced. Thus, $m_{3}$ can be assumed to be naturally small. Besides, a large $m_{3}$ can also give rise to significant mixing between the two Higgs doublets, which is strictly constrained from the present Higgs data.

\section{A. Neutrino mass generation}

The neutrino oscillation data $[3,51,52]$ indicate that at least two of the three light neutrinos have nonzero mass. One of the most natural ways to generate tiny neutrino mass is via the seesaw mechanism [4-12]. In $\nu$ HDM the mechanism is very similar to that of the Type-I seesaw [4-7]. The mixing between light and heavy neutrinos is introduced via the term $y_{\nu} \bar{L} \phi_{\nu} N$ in the aftermath of symmetry breaking, when $\phi_{\nu}$ gets a VEV. In the basis $\{\nu, N\}$ the $6 \times 6$ neutrino mass matrix looks like

$$
\mathcal{M}_{6 \times 6}=\left(\begin{array}{cc}
0_{3 \times 3} & m_{D 3 \times 3} \\
m_{D 3 \times 3}^{T} & m_{R 3 \times 3}
\end{array}\right),
$$

where $m_{D}=y_{\nu} v_{\nu}$. The light effective $3 \times 3$ neutrino mass matrix in the approximation $m_{D} \ll m_{R}$ is given by

$$
M_{\nu}=m_{D} m_{R}^{-1} m_{D}^{T}
$$

The above equation looks exactly similar to what we obtain in the canonical Type-I seesaw scenario. The only difference is that in the present framework $v_{\nu}$ can be quite small and as a result one can have larger $y_{\nu}$ compared to the canonical Type-I seesaw scenario, thus making this model phenomenologically more interesting. In order to fit the oscillation data, one also needs to account for the mixing among the three light neutrino states constrained by the Pontecorvo-Maki-Nakagawa-Sakata (PMNS) matrix. One can rewrite $M_{\nu}$ in Eq. (10) as

$$
M_{\nu}=U^{T} m_{\nu}^{\text {diag }} U
$$

where $m_{\nu}^{\text {diag }}$ is the diagonal light $3 \times 3$ neutrino mass matrix and $U$ is the PMNS mixing matrix. In order to produce proper mixing satisfying the experimental bounds on the PMNS matrix elements, one of the matrices, $m_{D}$ or $m_{R}$, has to be off-diagonal. Here we choose to keep $m_{R}$ diagonal and fit the PMNS matrix via an off-diagonal $m_{D}$. Thus $y_{\nu}$ is obtained using Casas-Ibarra parametrization [53]

$$
y_{\nu}=\frac{1}{v_{\nu}} \sqrt{m_{R}^{\text {diag }}} R \sqrt{m_{\nu}^{\text {diag }}} U^{T},
$$

where $R$ can be any orthogonal matrix and complex provided $R^{T} R=1$. For simplicity, we have chosen $R$ to be an identity matrix. 
Thus with correct choices of the parameters $m_{D}$ and $m_{R}$, Eq. (9) is capable of explaining the neutrino oscillation data at the tree level itself. There is a potential source of large correction $[54,55]$ to the neutrino states at one loop arising from the $H_{\nu}\left(A_{\nu}\right)$ loops. These mass corrections can be sizable enough to violate the experimental limits. However, the loop contributions to the neutrino masses corresponding to $H_{\nu}$ and $A_{\nu}$ have a mutual sign difference and can exactly cancel each other if they are mass degenerate [37,56-58]. As can be seen from both Eqs. (7) and (8), the mass splitting between these two states is driven by the parameter $\lambda_{5}$ which is therefore set equal to zero throughout this work.

\section{CONSTRAINTS AND BENCHMARK POINTS}

Constraints on the charged Higgs mass and its couplings may arise from direct collider search results, neutrino oscillation data, and lepton flavor violating decay branching ratios. The LHC Collaborations have looked for signatures of exotic scalars in various channels and put bounds on the charged Higgs mass in the range 300$1000 \mathrm{GeV}$ provided it can decay only into a top and a bottom quark [59-62]. However, in our present scenario, the charged Higgs, being a neutrinophilic one, does not couple to the quarks. In such scenarios, there are no direct search constraints on $m_{H_{\nu}^{ \pm}}$. In principle, the constraints derived from slepton searches at the LHC can be reinterpreted to put bounds on the neutrinophilic charged Higgs masses although only in the massless limit of the lightest neutralino. Two body decay of the sleptons into a charged lepton and lightest neutralino gives rise to a dilepton signal which can be relevant for the present scenario. Existing data exclude slepton masses up to $450 \mathrm{GeV}$ in the presence of a massless neutralino [63,64]. However, one always obtains same-flavor-opposite-sign (SFOS) lepton pairs from such slepton pair production processes. The signal requirement also demands a jet veto in the central region alongside the SFOS lepton pair for such analyses. In the present scenario, the largest event rate in such a signal region can be obtained when $H_{\nu}^{ \pm}$decays into a charged lepton and a heavy neutrino. A heavy neutrino further decays into a light neutrino and Z-boson which further decays invisibly. Clearly, the resulting signal cross section is rendered small due to branching suppressions. The demand of SFOS lepton pairs makes this cross section even smaller. ${ }^{3}$ Thus, the existing slepton mass limit when reinterpreted for $m_{H_{\nu}^{ \pm}}$proves to be much weaker. Its couplings with the heavy neutrinos, on the other hand, can be constrained from neutrino oscillation data and lepton flavor violating decay branching ratios [44]. As mentioned in Sec. II A, we have used off-diagonal $m_{D}$ while fitting the PMNS matrix. These

\footnotetext{
${ }^{3}$ The obtained signal cross section for our lightest benchmark point even before the detector simulation is less than the observed number as quoted in $[63,64]$.
}

off-diagonal entries are severely constrained from lepton flavor violation (LFV) decay branching ratio constraints [65-71]. These constraints are also reflected upon our choice of the neutrinophilic Higgs VEV, $v_{\nu}$. It has been observed and also verified by us that $v_{\nu}$ can be $\sim 10^{-2} \mathrm{GeV}$ [44] at the smallest, if the neutrino oscillation data and the LFV constraints are to be satisfied simultaneously, the most stringent constraint arising from the nonobservation of $\mathrm{BR}(\mu \rightarrow e \gamma)[65,66]$. This constraint puts the spontaneously breaking $\mathcal{Z}_{2}$ scenario in jeopardy. As evident from Fig. 2, such a choice of $v_{\nu}$ is clearly ruled out from restrictions on $\mathrm{CMB}$ temperature anisotropies induced by domain walls. However, if the $\mathcal{Z}_{2}$ symmetry is broken explicitly, this domain wall problem can be averted. Hence for this work, we choose to work with the $m_{3} \neq 0$ scenario only.

\section{A. Charged Higgs branching ratios and pair production cross section}

The possible decay modes of the neutrinophilic charged Higgs $\left(H_{\nu}^{ \pm}\right)$in our present scenario are $H_{\nu}^{ \pm} \rightarrow N \ell^{ \pm}$, $H_{\nu}^{ \pm} \rightarrow N \tau^{ \pm}, H_{\nu}^{ \pm} \rightarrow H_{\nu} W^{ \pm}$, and $H_{\nu}^{ \pm} \rightarrow A_{\nu} W^{ \pm}$. The relevant interaction vertices are given in Appendix A. Depending on the mass hierarchy of $H_{\nu}^{ \pm}, H_{\nu}\left(A_{\nu}\right)$, and $N$ and the choice of neutrino mass hierarchy one (or two) of these decay modes determines the event rates of the different possible final states at the collider. Note that the branching ratios of the decays into the neutral $C P$-even and $C P$-odd Higgs states are always the same since they are mass degenerate by our choice of the parameters. These two decay modes dominate over the heavy neutrino decay modes always, if the mass difference, $\Delta m=$ $m_{H_{\nu}^{ \pm}}-m_{H_{\nu}}\left(m_{A_{\nu}}\right)$, is larger than that of the $W$-boson mass, $m_{W}$. This is an artifact of the small Dirac neutrino Yukawa parameters, which are otherwise constrained by neutrino oscillation data and the nonobservation of LFV decays. The $y_{\nu}$ being smaller by orders of magnitude from the competitive gauge coupling, a large branching ratio into the $N \ell^{ \pm}$or $N \tau^{ \pm}$decay modes is not ensured even if $\Delta m<m_{W}$. In spite of the additional phase space suppression, threebody decays of $H_{\nu}^{ \pm}$via off-shell $W$-decay, dominate over these two-body modes unless $\Delta m \ll m_{W}$. This behavior is depicted in Fig. 3 where the competitive nature of $\operatorname{BR}\left(H_{\nu}^{ \pm} \rightarrow N \ell^{ \pm}\right)$and $\operatorname{BR}\left(H_{\nu}^{ \pm} \rightarrow H_{\nu}\left(A_{\nu}\right) \ell \nu\right)$, where $\ell=e$, $\mu$, is clearly visible through the distributions of the starred and circular points, respectively. $\operatorname{BR}\left(H_{\nu}^{ \pm} \rightarrow N \ell^{ \pm}\right)$overtakes the three-body decay branching ratio only if $\Delta m<50 \mathrm{GeV}$. For $\Delta m>m_{W}, \operatorname{BR}\left(H_{\nu}^{ \pm} \rightarrow H_{\nu}\left(A_{\nu}\right) W^{ \pm}\left(\rightarrow \ell^{ \pm} \nu\right)\right)$ takes over and remains the only dominant decay mode.

In Fig. 4, we have shown variation of the $H_{\nu}^{ \pm}$production cross section at the LHC and an $e^{+} e^{-}$collider. The figure on the left shows the variation of the cross sections as a function of $m_{H_{\nu}^{ \pm}}$at the $13 \mathrm{TeV}$ LHC and different center-of-mass energies ( $500 \mathrm{GeV}, 1 \mathrm{TeV}$, and $3 \mathrm{TeV})$ at an 


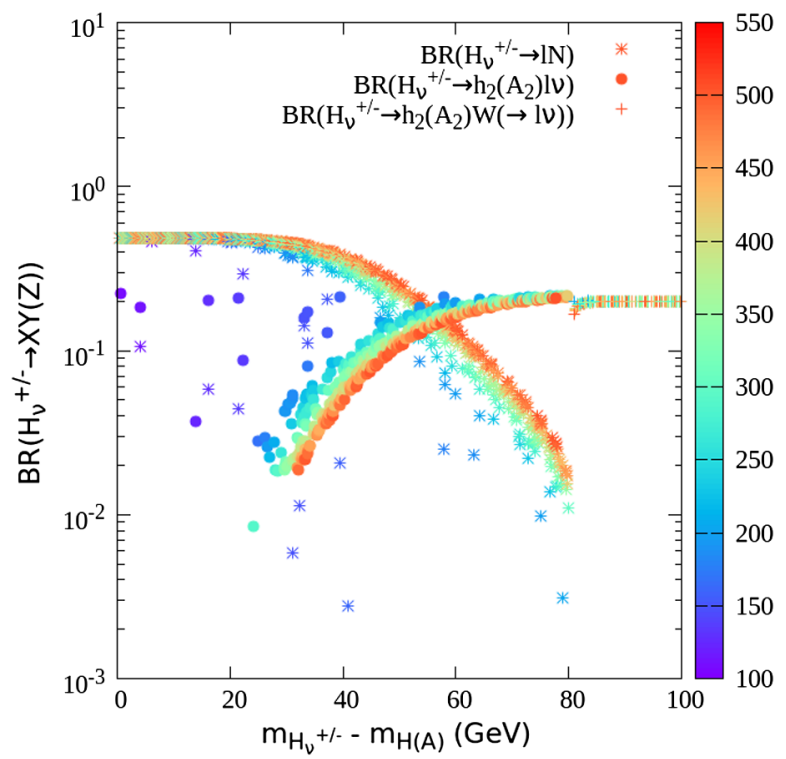

FIG. 3. Variation of $\operatorname{BR}\left(H_{\nu}^{ \pm} \rightarrow N \ell^{ \pm}\right), \operatorname{BR}\left(H_{\nu}^{ \pm} \rightarrow H_{\nu}\left(A_{\nu}\right) \times\right.$ $\left.W^{ \pm}\left(\rightarrow \ell^{ \pm} \nu\right)\right)$, and $\mathrm{BR}\left(H_{\nu}^{ \pm} \rightarrow H_{\nu}\left(A_{\nu}\right) \ell \nu\right)$ as a function of $\Delta m$. The color coded bar on the right shows the variation of $m_{H_{\nu}^{ \pm}}$. For all the points, $m_{N}$ is kept fixed at $100 \mathrm{GeV}$. Normal hierarchy is assumed for the light neutrinos. For inverted hierarchy, although the numerical values of the BRs are expected to be different, the pattern of the distribution remains the same.

$e^{+} e^{-}$collider. Note that, at the LHC, the $H_{\nu}^{ \pm}$production channels include $p p \rightarrow H_{\nu}^{ \pm} H_{\nu}^{\mp}, p p \rightarrow H_{\nu}^{ \pm} H_{\nu}$, and $p p \rightarrow$ $H_{\nu}^{ \pm} A_{\nu}$ while for the $e^{+} e^{-}$collider, pair production is the only viable option. Since we have assumed $m_{H_{\nu}}=m_{A_{\nu}}$ for our study, the cross sections of the above mentioned second and third production channels are exactly equal. Hence we have shown their combined cross section in the figure, and evidently, it dominates over the pair production cross section throughout the entire charged Higgs mass range. However, both these cross sections fall rapidly with increasing mass. On the other hand, at an $e^{+} e^{-}$collider the cross section falls far less rapidly implying the fact that such a collider will be more effective than the LHC in order to probe heavier charged Higgs masses. The figure on the right shows the variation of the pair production cross section at an $e^{+} e^{-}$collider with varying center-of-mass energies for our chosen benchmark points. Moreover, a lepton collider is likely to be much cleaner in terms of the SM background contributions. In this work, we have taken into account all the aforementioned production channels for LHC and just the pair production for the $e^{+} e^{-}$collider analysis.

\section{B. Choice of benchmark points}

We now proceed to choose some benchmark points representing the different interesting features of the present scenario for further collider studies. As discussed earlier, one can obtain different possible final states depending upon the mass hierarchies of $H_{\nu}^{ \pm}, H_{\nu}\left(A_{\nu}\right)$, and $N$. Since we also aim to correlate the light neutrino mass hierarchy with the multiplicity of different lepton flavor final states, we will study cases in which at least one of the heavy neutrinos is lighter than the neutrinophilic Higgs states so that it can appear in the cascade. In Table I we present the input

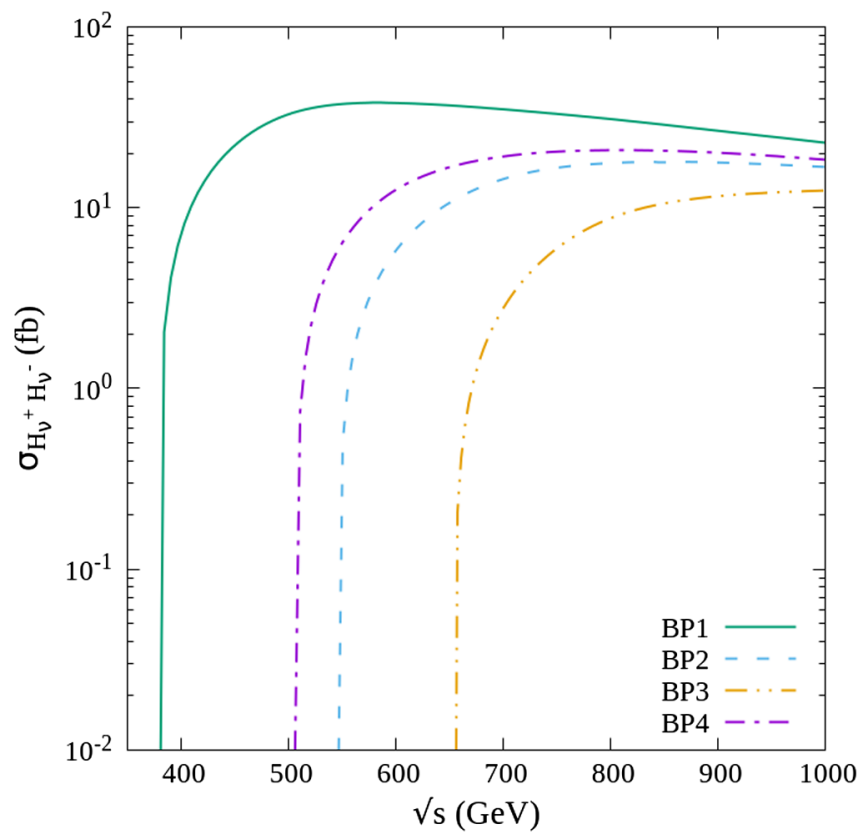

FIG. 4. Variation of the charged Higgs pair production cross section at the LHC and an $e^{+} e^{-}$collider at center-of-mass energies of 13 and $1 \mathrm{TeV}$, respectively. The distribution on the right shows variation of the charged Higgs pair production cross section at an $e^{+} e^{-}$ collider with varying center-of-mass energy $(\sqrt{s})$ for our four benchmark points. 
TABLE I. Relevant model parameters and masses. As mentioned before the parameter $\lambda_{5}$ is set equal to zero throughout this work.

\begin{tabular}{|c|c|c|c|c|c|c|c|c|c|c|c|c|}
\hline Parameters & \multicolumn{3}{|c|}{ BP1 } & \multicolumn{3}{|c|}{ BP2 } & \multicolumn{3}{|c|}{ BP3 } & \multicolumn{3}{|c|}{ BP4 } \\
\hline$\lambda_{1}$ & \multicolumn{3}{|c|}{0.270} & \multicolumn{3}{|c|}{0.210} & \multicolumn{3}{|c|}{0.235} & \multicolumn{3}{|c|}{0.212} \\
\hline$\lambda_{2}$ & \multicolumn{3}{|c|}{0.50} & \multicolumn{3}{|c|}{0.50} & \multicolumn{3}{|c|}{0.50} & \multicolumn{3}{|c|}{0.50} \\
\hline$\lambda_{3}$ & \multicolumn{3}{|c|}{1.50} & \multicolumn{3}{|c|}{1.50} & \multicolumn{3}{|c|}{1.50} & \multicolumn{3}{|c|}{1.50} \\
\hline$\lambda_{4}$ & \multicolumn{3}{|c|}{-0.01} & \multicolumn{3}{|c|}{-1.50} & \multicolumn{3}{|c|}{-0.01} & \multicolumn{3}{|c|}{-1.10} \\
\hline$m_{3}^{2} \mathrm{GeV}^{2}$ & \multicolumn{3}{|c|}{-1.50} & \multicolumn{3}{|c|}{-1.50} & \multicolumn{3}{|c|}{-4.50} & \multicolumn{3}{|c|}{-1.50} \\
\hline$m_{R}^{i i}[\mathrm{GeV}]$ & \multicolumn{3}{|c|}{100.0} & \multicolumn{3}{|c|}{100.0} & \multicolumn{3}{|c|}{200.0} & \multicolumn{3}{|c|}{125.0} \\
\hline$m_{H_{\nu}}, m_{A_{\nu}}[\mathrm{GeV}]$ & \multicolumn{3}{|c|}{187.5} & \multicolumn{3}{|c|}{187.9} & \multicolumn{3}{|c|}{325.6} & \multicolumn{3}{|c|}{188.5} \\
\hline$m_{H_{\nu}^{ \pm}}[\mathrm{GeV}]$ & \multicolumn{3}{|c|}{188.5} & \multicolumn{3}{|c|}{272.8} & & 326.4 & & & 252.8 & \\
\hline$m_{N}[\mathrm{GeV}]$ & & 100.0 & & & 100.0 & & & 200.0 & & & 125.0 & \\
\hline & & (Normal & & & Normal & & & (Normal) & & & (Normal & \\
\hline & ( 1.445 & 2.261 & $0.336)$ & ( 1.445 & 2.261 & $0.336)$ & ( 2.044 & 3.197 & $0.476)$ & ( 1.616 & 2.528 & $0.376)$ \\
\hline$y_{\nu}$ & 2.261 & 5.719 & 3.396 & 2.261 & 5.719 & 3.396 & 3.197 & 8.088 & 4.802 & 2.528 & 6.394 & 3.797 \\
\hline & 0.336 & 3.396 & $6.944)$ & 0.336 & 3.396 & $6.944)$ & 0.476 & 4.802 & $9.820)$ & 0.376 & 3.797 & $7.763)$ \\
\hline & & (Invertec & & & Inverted & & & Inverted & & & Inverted & \\
\hline$\left(\times 10^{3}\right)$ & 3.796 & 7.116 & $5.594)$ & 3.796 & 7.116 & $5.594)$ & 5.369 & 10.060 & $8.053)$ & 4.245 & 7.956 & $6.367)$ \\
\hline & 7.116 & 0.785 & 2.135 & 7.116 & 0.785 & 2.135 & 10.060 & 1.109 & 3.019 & 7.956 & 0.877 & 2.387 \\
\hline & 5.694 & 2.135 & $3.101)$ & 5.694 & 2.135 & $3.101)$ & 8.053 & 3.019 & $4.386)$ & 6.367 & 2.387 & $3.467)$ \\
\hline
\end{tabular}

parameters, the relevant masses, and the resulting $y_{\nu}$ for the four benchmark points of our choice. We have incorporated the complete model in SARAH [72-76], and subsequently imported in SPheno [77,78] in order to perform the analytical and numerical computation of the masses and mixings of the particles, their branching ratios, and other relevant constraints. See Appendix B for LFV constraints for our benchmarks.

The four benchmark points are chosen such that all the dominant decay modes of the neutrinophilic Higgs and the heavy neutrinos are highlighted by different mass hierarchies. The relevant branching ratios are shown in Table II. The two most dominant decay modes of $H_{\nu}^{ \pm}$are $N \ell$, where $\ell=e, \mu, \tau$, and $H_{\nu}\left(A_{\nu}\right) W^{ \pm}$. The first decay mode is driven by the Dirac neutrino Yukawa couplings, $y_{\nu}$, whereas the second one is driven by gauge couplings. As discussed above, the elements of $y_{\nu}$ are already constrained from the neutrino oscillation data as well as from the LFV constraints, and thus are in general weaker than the competitive gauge coupling. Hence, if the mass splittings among the neutral and charged neutrinophilic Higgs and the heavy neutrino states are such that both $N \ell$ and $H_{\nu}\left(A_{\nu}\right) W^{ \pm}$decay modes are kinematically accessible for $H_{\nu}^{ \pm}$, the gauge boson associated one becomes its only relevant decay mode. However, if at least one of the heavy neutrinos is lighter than the $H_{\nu}^{ \pm}$and the $H_{\nu}\left(A_{\nu}\right)$ states are almost degenerate to it, then the decay via heavy neutrinos becomes important. The latter scenario is highlighted in BP1 and BP3 while BP2 represents the former scenario. BP4, on the other hand, highlights the situation where the

TABLE II. Relevant branching ratios. Here $\ell=e, \mu$.

\begin{tabular}{|c|c|c|c|c|c|c|c|c|}
\hline \multirow[b]{2}{*}{ Branching ratio } & \multicolumn{2}{|c|}{ BP1 } & \multicolumn{2}{|c|}{ BP2 } & \multicolumn{2}{|c|}{ BP3 } & \multicolumn{2}{|c|}{ BP4 } \\
\hline & Normal & Inverted & Normal & Inverted & Normal & Inverted & Normal & Inverted \\
\hline $\mathrm{BR}\left(H_{\nu}^{ \pm} \rightarrow N \ell^{ \pm}\right)$ & 0.49 & 0.77 & $\cdots$ & $\cdots$ & 0.49 & 0.77 & 0.05 & 0.13 \\
\hline $\operatorname{BR}\left(H_{\nu}^{ \pm} \rightarrow N \tau^{ \pm}\right)$ & 0.51 & 0.23 & $\cdots$ & $\cdots$ & 0.51 & 0.23 & 0.06 & 0.04 \\
\hline $\operatorname{BR}\left(H_{\nu}^{ \pm} \rightarrow H_{\nu} W^{ \pm}\right)$ & $\ldots$ & $\ldots$ & 0.50 & 0.50 & $\cdots$ & $\cdots$ & $\ldots$ & $\ldots$ \\
\hline $\operatorname{BR}\left(H_{\nu}^{ \pm} \rightarrow A_{\nu} W^{ \pm}\right)$ & $\cdots$ & $\cdots$ & 0.50 & 0.50 & $\cdots$ & $\cdots$ & $\cdots$ & $\cdots$ \\
\hline $\mathrm{BR}\left(H_{\nu}^{ \pm} \rightarrow H_{\nu} \ell \nu\right)$ & $\ldots$ & $\ldots$ & $\ldots$ & $\ldots$ & $\ldots$ & $\ldots$ & 0.10 & 0.09 \\
\hline $\operatorname{BR}\left(H_{\nu}^{ \pm} \rightarrow A_{\nu} \ell \nu\right)$ & $\ldots$ & $\cdots$ & $\ldots$ & $\ldots$ & $\ldots$ & $\cdots$ & 0.10 & 0.09 \\
\hline $\operatorname{BR}\left(N \rightarrow \ell^{+} W^{-}\right)$ & 0.21 & 0.43 & 0.21 & 0.43 & 0.16 & 0.21 & 0.17 & 0.23 \\
\hline $\operatorname{BR}\left(N \rightarrow \ell^{-} W^{+}\right)$ & 0.21 & 0.43 & 0.21 & 0.43 & 0.16 & 0.21 & 0.17 & 0.23 \\
\hline $\operatorname{BR}\left(N \rightarrow \tau^{+} W^{-}\right)$ & 0.23 & 0.01 & 0.23 & 0.01 & 0.18 & 0.13 & 0.20 & 0.14 \\
\hline $\operatorname{BR}\left(N \rightarrow \tau^{-} W^{+}\right)$ & 0.23 & 0.01 & 0.23 & 0.01 & 0.18 & 0.13 & 0.20 & 0.14 \\
\hline $\mathrm{BR}\left(N \rightarrow \nu_{\ell} Z\right)$ & 0.12 & 0.12 & 0.12 & 0.12 & 0.32 & 0.32 & 0.27 & 0.27 \\
\hline$\underline{\operatorname{BR}\left(H_{\nu}\left(A_{\nu}\right) \rightarrow \nu_{\ell} N\right)}$ & 1.00 & 1.00 & 1.00 & 1.00 & 1.00 & 1.00 & 1.00 & 1.00 \\
\hline
\end{tabular}




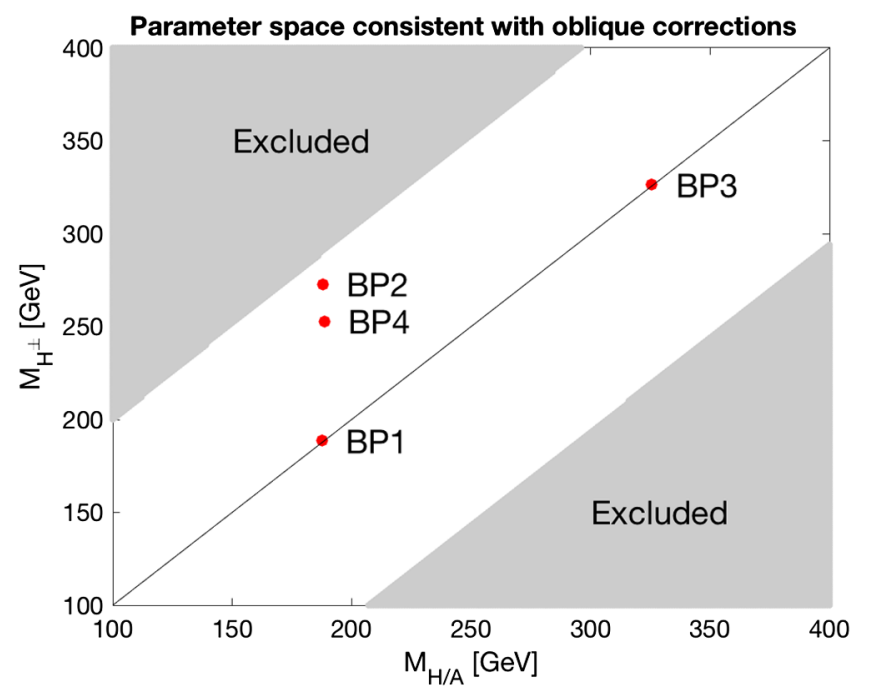

FIG. 5. In the gray region, the oblique corrections induced by the neutrinophilic Higgses are too large. A too large mass difference $\Delta m \equiv m_{H_{\nu}^{ \pm}}-m_{H_{\nu}, A_{\nu}}$ is disfavored. Red dots label the chosen benchmark points. The black line corresponds to $m_{H_{\nu}^{ \pm}}=m_{H_{\nu}, A_{\nu}}$.

two-body mode $\ell N$ competes with the three-body decay into $H_{\nu}$ (or $A_{\nu}$ ) alongside an off-shell $W$-boson. However, $\Delta m$ being on the larger side, the three-body decay dominates as discussed earlier in Sec. III A. The heavy neutrinos $(N)$ in this scenario can decay either via the SM gauge bosons $\left(W^{ \pm}, Z\right)$ or the different Higgs states. Note that decays of $N$ into $W^{ \pm}, Z$, and $h$ can occur only through their mixing with the light neutrinos which are suppressed in the present scenario. Hence, these decay modes become relevant for $N$ only if the neutrinophilic Higgs states are kinematically inaccessible to it. The choice of neutrino mass hierarchy clearly reflects in the branching ratios of both $H_{\nu}^{ \pm}$and $N$ and is also expected to be reflected in the final event rates of the multilepton signals we intend to explore.

In addition, we checked the effect of neutrinophilic Higgses on the oblique parameters $(S, T, U)$. We have ensured the corrections induced by our benchmark points do not exceed the uncertainties given in [79]. See Fig. 5 for the allowed $\left(m_{H_{\nu}, A_{\nu}}, m_{H_{\nu}^{ \pm}}\right)$values.

\section{COLLIDER ANALYSIS}

Charged Higgs in our benchmark scenarios can give rise to novel signatures in lepton enriched final states. Majorana neutrinos, if produced via cascade from the charged Higgs can further decay resulting in same-sign leptonic final states, which are characteristic to seesaw models and also have much less SM background. The gauge bosons resulting from the decays of the neutrinophilic Higgs and heavy neutrinos may also decay leptonically, and thus one can easily obtain a multilepton final state associated with missing energy.
Leptonic branching ratios of the gauge bosons being small, one would expect smaller event rates in the final state with increasing lepton multiplicity. However, it also means less SM background to deal with resulting in cleaner signals. In this section we explore the different possible multilepton final states with or without the presence of additional jets along with detailed signal to background simulation in order to ascertain the discovery potential of the charged Higgs for our chosen benchmark points in the context of $13 \mathrm{TeV}$ LHC as well as future lepton colliders.

\section{A. Identifying signal regions}

In the context of LHC, we aim to study cleaner multilepton channels with no tagged jets in the final state. The possible final states that we probe in the present context are $\geq 6 \ell+\mathbb{E}_{T}+X(\mathbf{S R} 1), \geq 5 \ell+\mathbb{E}_{T}+X$ (SR2), and samesign trilepton $(S S 3 \ell)+\mathbb{E}_{T}+X(\mathbf{S R 3 S R 3})$, where $X$ represents everything else (jets, photons, or leptons) ${ }^{4}$ in the final states. As mentioned earlier, the various branching ratios of $H_{\nu}^{ \pm}$and hence the final signal event rates depend on the mass difference factor $\Delta m$. Thus, it is interesting to study how the signal rates vary depending on $\Delta m$ which in turn can also provide an indirect hint about the masses of $H_{\nu}$ and (or) $A_{\nu}$.

In Fig. 6 we have shown the variation of the cross sections corresponding to the three signal regions mentioned above as a function of $\Delta m$ with color-coded $m_{H_{\nu}^{ \pm}}$. Note that these cross sections are theoretical estimates obtained after combining contributions from all three relevant production modes of $H_{\nu}^{ \pm}$at the LHC prior to detector simulation and do not include the cut efficiencies. The two rows of figures correspond to normal and inverted hierarchies of neutrino masses, respectively. While SR1 only receives a contribution from pair production, both SR2 and SR3 are enriched with contributions from pair production as well as associated production of the $H_{\nu}^{ \pm}$. Most of the signal events corresponding to SR1 and SR2 are expected to arise from $H_{\nu}^{ \pm}$decay into a charged lepton and a heavy neutrino followed by the heavy neutrino decay into a charged lepton and $W$. Depending on the leptonic or hadronic decays of the $W$-bosons, one can obtain various lepton multiplicities as represented by these signal regions. The signal cross sections are largest when $H_{\nu}^{ \pm}$and $H_{\nu}\left(A_{\nu}\right)$ are mass degenerate for any given $m_{H_{\nu}^{ \pm}}$, and they drop with increasing $m_{H_{\nu}^{ \pm}}$and $\Delta m$. SR3, on the other hand, receives more contributions when $H_{\nu}^{ \pm}$decays into $H_{\nu}$ (or $A_{\nu}$ ) along with an on-shell or off-shell $W$-boson. Moreover, in the case of pair production, same-sign leptons cannot be obtained if both the $H_{\nu}^{ \pm}$decays via $\ell^{ \pm} N$ result in the cross section of $\mathbf{S R 3}$ being smaller with smaller $\Delta m$. However, the associated production channels contribute dominantly to this signal region throughout the whole

${ }^{4}$ For SR3, $X$ consists of no leptons since in this case, we demand exactly three leptons with the same sign in the final state. 

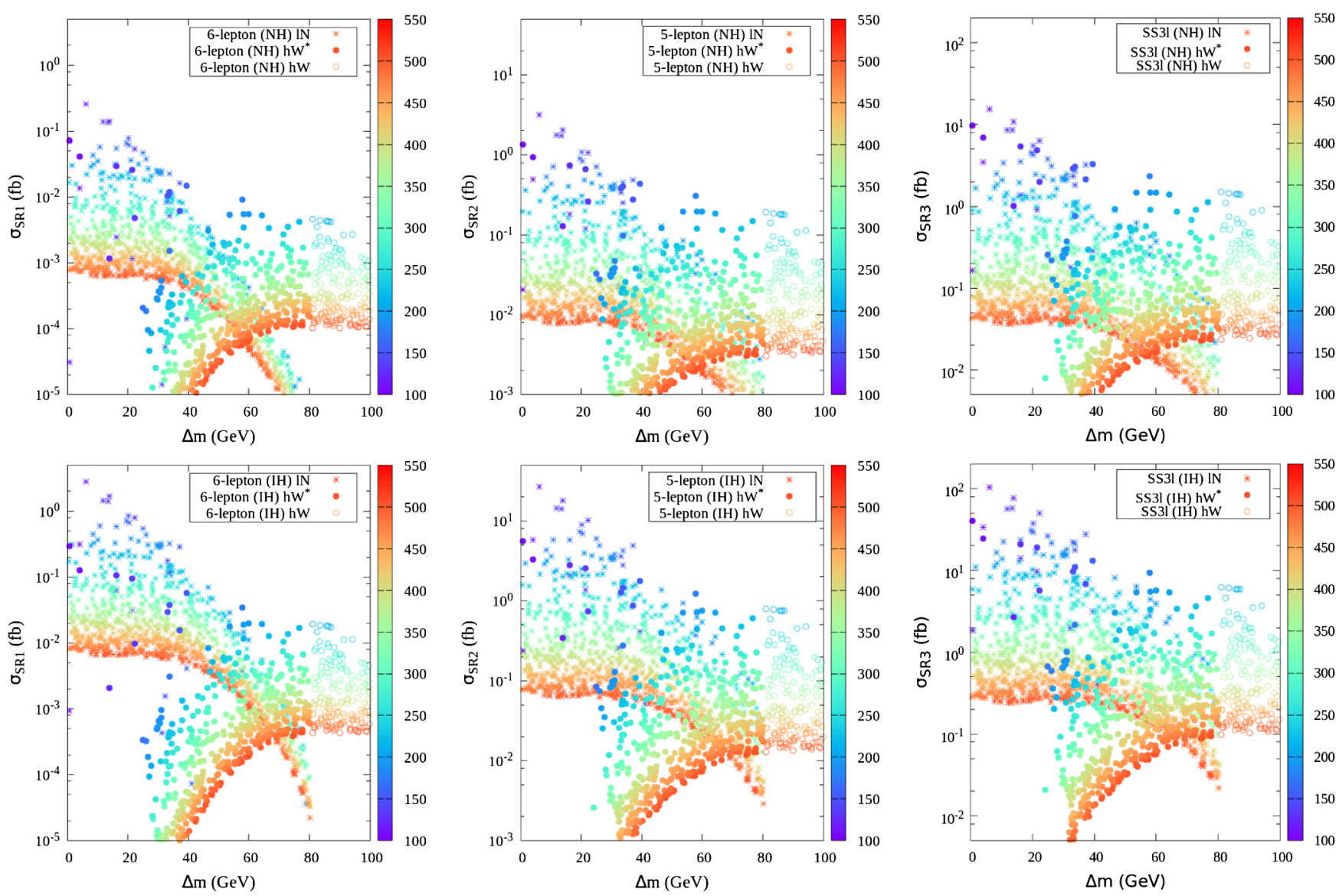

FIG. 6. Variation of cross sections corresponding to the signal regions SR1, SR2, and SR3 as a function of $\Delta m$ at 13 TeV LHC. The two rows represent scenarios with normal and inverted hierarchies of the light neutrino masses, respectively. The color coding represents variation of $m_{H_{\nu}^{ \pm}}$. For all points, heavy neutrino masses are kept at $100 \mathrm{GeV}$.

range of $\Delta m$. As evident from Fig. 6, SR3 is the most favorable channel to look for such scenarios. In general, the inverted hierarchy of the light neutrino masses is expected to generate more multileptonic events owing to the larger branching ratio, $\operatorname{BR}\left(H_{\nu}^{ \pm} \rightarrow e N\right)$, as reflected by the plots on the bottom row.

In a similar way, we now proceed to choose some signal regions for our analyses in the context of an $e^{+} e^{-}$collider. The possible final states we probe in this context are $\geq 5 \ell+\not E+X$ (SR4), $\geq 4 \ell+\geq 2-$ jet $+\not E+X$ (SR5), and $S S 3 \ell+\not E+X$ (SR6). ${ }^{5}$ The corresponding signal rates are showcased as a function of $\Delta m$ in Fig. 7. Trends of the distributions are similar to what we obtained for the LHC case. However, the difference in the production cross section is manifested by the signal cross sections indicating a larger event rate at the LHC for similar final states at the low $m_{H_{\nu}^{ \pm}}$region. The rapid fall in production cross section with increasing $m_{H_{\nu}^{ \pm}}$at the LHC makes it less relevant for heavier charged Higgs masses. An $e^{+} e^{-}$collider can be more effective provided the center-of-mass energy is large

${ }^{5}$ Just as SR3, $X$ in SR6 does not contain any leptons. enough for the production. Here, the signal rates drop alarmingly close to $m_{H_{\nu}^{ \pm}} \sim 500 \mathrm{GeV}$ due to the choice of center-of-mass energy as $1 \mathrm{TeV}$.

\section{B. Analysis}

In order to carry out the simulation, events were generated at the parton level using MADGRAPH5 [80,81] with NN23LO1 parton distribution function $[82,83]$ and the default dynamic factorization and renormalization scales [84]. We have used PYTHIA [85] for the subsequent decay of the particles, showering, and hadronization. After that the events are passed through DelPHES [86-88] for detector simulation. Jets have been reconstructed using the anti-kT algorithm via FASTJET $[89,90]$. The $b$-jet and $\tau$-jet tagging efficiencies as well as the mistagging efficiencies of the light jets as $b$ - or $\tau$-jet have been incorporated according to the latest ATLAS studies in this regard [91].

\section{Primary selection criteria}

We have applied the following cuts (CO) on the jets, leptons, and photons in order to identify them as final state particles: 

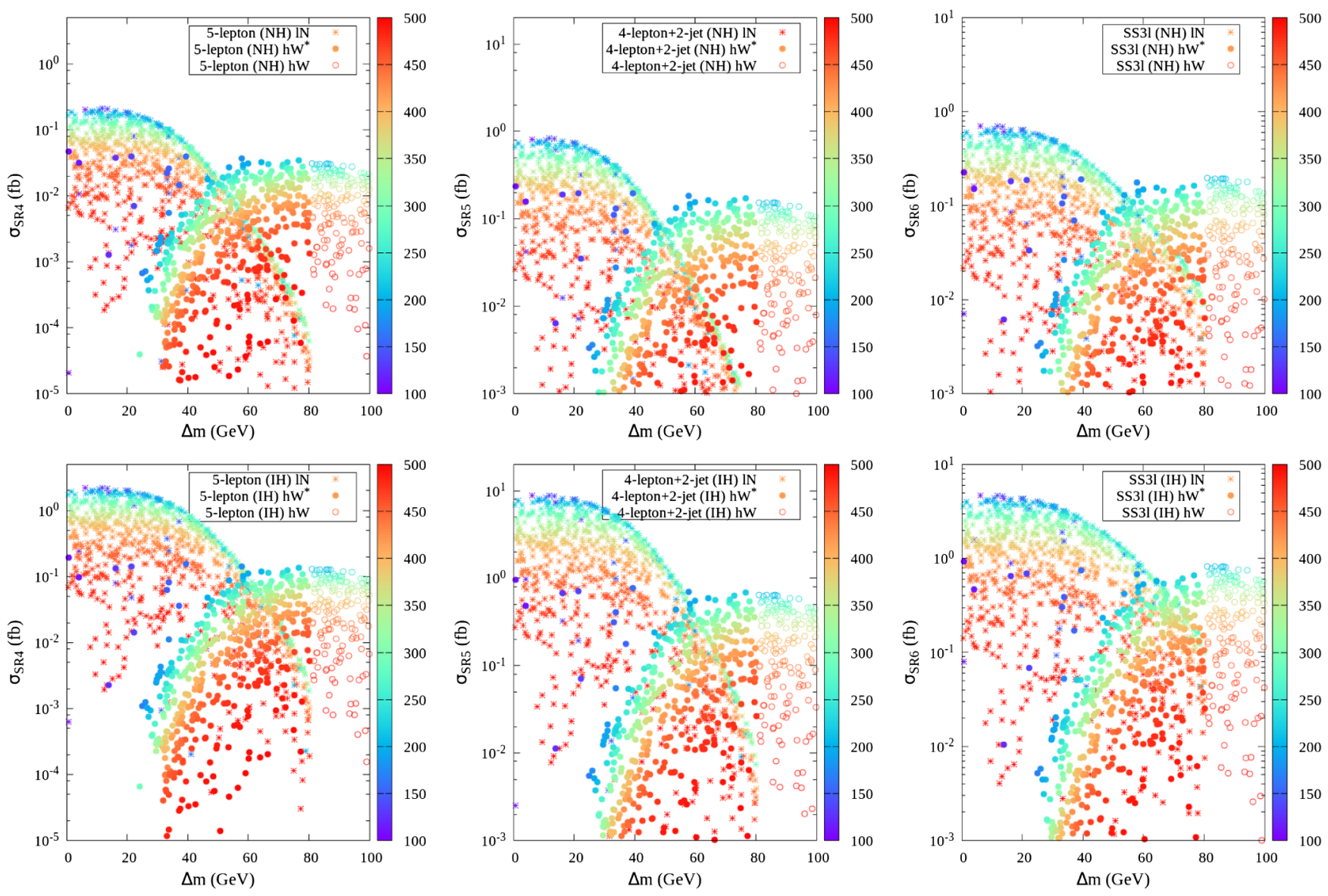

FIG. 7. Variation of cross sections corresponding to the signal regions SR4, SR5, and SR6 as a function of $\Delta m$ at an $e^{+} e^{-}$ collider with $1 \mathrm{TeV}$ center-of-mass energy. The two rows represent scenarios with normal and inverted hierarchies of the light neutrino masses, respectively. The color coding represents variation of $m_{H_{\nu}^{ \pm}}$. For all points, heavy neutrino masses are kept at $100 \mathrm{GeV}$.

(i) All the charged leptons are selected with a transverse momentum threshold $p_{T}^{\ell}>10 \mathrm{GeV}$ and in the pseudorapidity window $|\eta|^{\ell}<2.5$.

(ii) All the jets including $b$-jets and $\tau$-jets must have $p_{T}^{j}>20 \mathrm{GeV}$ and $|\eta|^{j}<2.5$.

(iii) We demand $\Delta R_{i j}>0.4$ between all possible pairs of the final state particles to make sure they are well separated.

As discussed in Sec. III, the choice of neutrino mass hierarchy affects the branching ratios of the neutrinophilic Higgs as well as the heavy neutrinos in certain flavor specific decay modes. Thus, the hierarchical effect is reflected by the abundance of a certain flavor of leptons in the signal events. As we have seen, one would expect less abundance of electrons in the final states for a normal hierarchy scenario compared to that for an inverted hierarchy. This feature is evident in Fig. 8 which shows the electron multiplicity in the final state with at least four leptons for BP1 in normal as well as inverted hierarchy

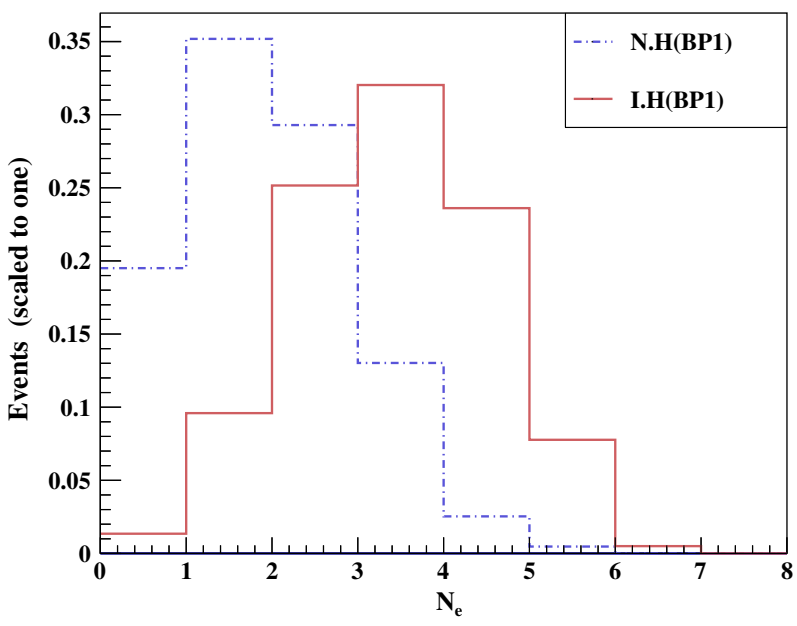

FIG. 8. Electron multiplicity distribution for BP1 in normal and inverted hierarchy scenarios indicated by blue and red lines respectively. The distributions correspond to the choice of final states as per SR2. 
TABLE III. Charged Higgs pair production cross sections and number of events corresponding to the three different signal regions at $1000 \mathrm{fb}^{-1}$ luminosity at $13 \mathrm{TeV}$ LHC for our chosen benchmark points.

\begin{tabular}{|c|c|c|c|c|c|}
\hline \multirow{2}{*}{$\begin{array}{l}\text { Benchmark } \\
\text { points }\end{array}$} & \multirow{2}{*}{$\begin{array}{c}\text { Production } \\
\text { cross section }[\mathrm{fb}] \\
(\sqrt{s}=13 \mathrm{TeV})\end{array}$} & \multirow{2}{*}{$\begin{array}{l}\text { Neutrino } \\
\text { hierarchy }\end{array}$} & \multicolumn{3}{|c|}{$\begin{array}{l}\text { Number of Events } \\
\left(\mathcal{L}=1000 \mathrm{fb}^{-1}\right)\end{array}$} \\
\hline & & & SR1 & SR2 & SR3 \\
\hline \multirow[t]{2}{*}{ BP1 } & 60.71 & Normal & 8 & 130 & 247 \\
\hline & & Inverted & 25 & 343 & 397 \\
\hline \multirow[t]{2}{*}{ BP2 } & 22.13 & Normal & $\ldots$ & 13 & 42 \\
\hline & & Inverted & 1 & 24 & 55 \\
\hline \multirow[t]{2}{*}{ BP3 } & 6.72 & Normal & 3 & 40 & 67 \\
\hline & & Inverted & 8 & 86 & 101 \\
\hline \multirow[t]{2}{*}{ BP4 } & 27.34 & Normal & 1 & 26 & 71 \\
\hline & & Inverted & 3 & 60 & 112 \\
\hline
\end{tabular}

scenarios. Such lepton multiplicity distributions can thus provide an indirect probe of the existing neutrino mass hierarchy.

\section{Results@LHC13}

In the context of LHC, we have studied the final states corresponding to SR1, SR2, and SR3 as defined in Sec. IV A. Although the choice of our signal regions ensure small or no SM background, we have checked the relevant production channels, $t \bar{t}, t \bar{t} Z\left(\gamma^{*}\right), t \bar{t} W, W W Z, W Z Z, Z Z Z$, and $Z Z+$ jets nevertheless in this regard. In Table III we show the expected number of different signal events at the $13 \mathrm{TeV}$ run of the LHC with an integrated luminosity $(\mathcal{L})$ of $1000 \mathrm{fb}^{-1}$ after imposing a transverse missing energy cut, $E_{T}>50 \mathrm{GeV}$ and $b$-jet veto $(\mathbf{C 1}){ }^{6}$ in addition to the primary selection criteria, C0. The choice of our signal regions combined with the cuts $\mathbf{C} 1$ render the SM backgrounds to negligible event numbers. We have observed that our SR1 is nearly backgroundless, whereas SR2 and SR3 are left with 2 and 1 SM-background events, respectively, at $1000 \mathrm{fb}^{-1}$ integrated luminosity. As for the obtained signal event numbers, one can easily get an estimate of the expected rate from Fig. 6 for the different final states. However, note that in these figures the heavy neutrino mass is kept fixed at $100 \mathrm{GeV}$ and if this mass is changed, so are the heavy neutrino branching ratios and hence the signal cross sections. However, the cross sections shown in these figures are good enough for order of magnitude estimation for a given $m_{H_{\nu}^{ \pm}}$.

As expected $\mathbf{S R} 1$ has the smallest event rate owing to its large lepton multiplicity, but with negligible SM background. Thus it can be a very clean signal but only if the charged Higgs mass is on the lighter side, as in BP1, and at least one of the heavy neutrinos is lighter than the charged

\footnotetext{
${ }^{6}$ These cuts help reduce some of the surviving SM background contributions.
}

Higgs. The situation, however, worsens considerably with increasing charged Higgs mass, as indicated by BP3. SR2 has a much better event rate and can probe $\mathbf{B P 1}$ and $\mathbf{B P 3}$ at much lower luminosity than SR1. As the numbers in Table III indicate, the inverted hierarchy scenario for BP1 can be probed with a $3 \sigma$ statistical significance at an integrated luminosity of $\sim 30 \mathrm{fb}^{-1}$ in both $\mathbf{S R 2}$ and SR3; i.e. if these signal regions are studied, $m_{H_{\nu}^{ \pm}}$in this mass range can be probed and possibly be excluded with the LHC data already accumulated. For the corresponding normal hierarchy case, however, for the same benchmark point, one needs $\mathcal{L} \sim 50 \mathrm{fb}^{-1}$ for similar discovery significance in SR3. BP3 requires an integrated luminosity of $\sim 100 \mathrm{fb}^{-1}$ (for inverted hierarchy) or more. For the benchmark points like BP4 and BP2, the decay $H_{\nu}^{ \pm} \rightarrow \ell N$ is either suppressed or absent altogether. Thus for such points SR1 ceases to be a viable signal region while SR2 is relevant only at large luminosities. In this case SR3 turns out to be the most viable signal region. In this signal region, to achieve $3 \sigma$ statistical significance in the inverted hierarchy case of BP4 and BP2 one requires $\mathcal{L} \sim 100 \mathrm{fb}^{-1}$ and $\sim 200 \mathrm{fb}^{-1}$, respectively. For all the benchmark points, the choice of light neutrino mass hierarchy is clearly manifested through the different signal event rates. Evidently, with multileptonic final states, an inverted hierarchy scenario is more likely to be probed at lower luminosities at the LHC.

\section{Results@ $e^{+} e^{-}$collider}

In Table IV we have presented the expected number of different signal events at the $1 \mathrm{TeV}$ run of an $e^{+} e^{-}$collider with an integrated luminosity of $100 \mathrm{fb}^{-1}$ after imposing a missing energy cut, $\mathbb{E}>50 \mathrm{GeV}$ and $b$-jet veto (D1), in addition to the primary selection criteria, C0. The event rates are quite good and devoid of any direct SM background, which makes it an ideal platform to look for a neutrinophilic charged Higgs. Although the number of

TABLE IV. Charged Higgs pair production cross sections and number of events corresponding to the three different signal regions at $100 \mathrm{fb}^{-1}$ luminosity at $1 \mathrm{TeV} e^{+} e^{-}$collider for our chosen benchmark points.

\begin{tabular}{lccrcr}
\hline \hline & \multirow{2}{*}{$\begin{array}{c}\text { Production } \\
\text { Benchmark }\end{array}$} & $\begin{array}{c}\text { Number of events } \\
\text { points }\end{array}$ & $(\sqrt{s}=13 \mathrm{TeV})$ & $\begin{array}{c}\text { Neutrino } \\
\left(\mathcal{L}=100 \mathrm{fb}^{-1}\right)\end{array}$ \\
\cline { 4 - 6 } BP1 & 22.83 & Normal & 36 & 47 & 9 \\
& \multirow{2}{*}{16.91} & Inverted & 77 & 90 & 9 \\
BP2 & \multirow{2}{*}{12.48} & Normal & 5 & 16 & 6 \\
& & Inverted & 6 & 23 & 8 \\
BP3 & \multirow{2}{*}{18.44} & Normal & 30 & 75 & 16 \\
& & Inverted & 63 & 122 & 17 \\
BP4 & Normal & 8 & 22 & 8 \\
& & Inverted & 16 & 39 & 12 \\
\hline \hline
\end{tabular}


events shown in Table IV correspond to $\mathcal{L}=100 \mathrm{fb}^{-1}$, the inverted hierarchy scenarios in $\mathbf{B P 1}$ and $\mathbf{B P 3}$ can be probed with a statistical significance of $3 \sigma$ at a much lower luminosity $\left(\sim 10 \mathrm{fb}^{-1}\right)$. Note the improved event rates in signal regions SR5 and SR6 despite the smaller $H_{\nu}^{ \pm}$pair production cross section in $\mathbf{B P 3}$ over those of BP1. This is a consequence of increased hadronic branching ratio of $N$ and improved cut efficiency due to the larger mass gap between $H_{\nu}^{ \pm}$and $N$ in BP3. Even BP2 which can be probed at the LHC only at very high luminosity can be probed here at around $\mathcal{L}=50 \mathrm{fb}^{-1}$ with similar statistical significance via SR5 which turns out to be the most favored signal in general for all the benchmark points. The overall signal rate is relatively weaker in SR6 due to better lepton tagging efficiency at a lepton collider, which results in a smaller number of events with exactly three same-sign leptons as demanded.

\section{SUMMARY AND CONCLUSIONS}

We have considered a simple extension of the SM with one additional scalar doublet and three generations of singlet right-handed Majorana neutrinos, where the additional Higgs states interact with the SM sector only via the right-handed neutrinos. The model, known as the neutrinophilic Higgs doublet model, is a well-motivated framework from the viewpoint of neutrino mass generation. The light neutrinos gain tiny nonzero masses via the Type-I seesaw mechanism when the neutrinophilic Higgs obtains a VEV to break the $\mathcal{Z}_{2}$ symmetry. We have discussed in brief why the spontaneous breaking of the $\mathcal{Z}_{2}$ symmetry is disfavored, if one imposes the constraints derived from the CMB temperature anisotropies induced by domain walls as well as LFV decay branching ratios. We have, therefore, considered a scenario where the $\mathcal{Z}_{2}$ parity is broken explicitly and thus is devoid of the domain wall problem. In such a scenario, the charged Higgs can have interesting collider phenomenology, explored in this work. Depending on the different decay modes of the neutrinophilic charged Higgs, we have identified some particularly clean signal regions likely to provide a hint of $\nu \mathrm{HDM}$ scenarios at the collider experiments.

We have also highlighted the interesting role play of the light neutrino mass hierarchy. Whether the neutrinos follow normal or inverted hierarchy is likely to be manifested via multiplicity of different flavored leptons in the final state. Thus such a finding at the collider experiments can complement the neutrino oscillation experiments which are yet to ascertain the correct mass hierarchy of the three light neutrinos.
The fact that the charged Higgs pair production cross section falls quite rapidly at the LHC with increasing mass led us to perform a comparative study between the LHC and a future $e^{+} e^{-}$machine in order to probe such scenarios. We observed that although LHC is quite efficient to probe light charged Higgs masses, an $e^{+} e^{-}$collider will be able to probe a much larger parameter space with heavier states.

\section{ACKNOWLEDGMENTS}

K. H. and S. M. acknowledge the H2020-MSCA-RICE2014 Grant No. 645722 (NonMinimalHiggs). T. K. expresses his gratitude to Magnus Ehrnrooth foundation for financial support. The work of S. K. R. was partially supported by funding available from the Department of Atomic Energy, Government of India, for the Regional Centre for Accelerator-based Particle Physics (RECAPP), Harish-Chandra Research Institute.

\section{APPENDIX A: RELEVANT INTERACTION VERTICES}

$$
\begin{array}{cc}
\ell^{\mp i} N^{j} H_{\nu}^{ \pm k}, & i \sum_{a=1}^{3} \sum_{b=1}^{3} y_{\nu}^{a b} U_{L}^{i a} U_{N}^{j 3+b}, \\
H_{\nu}^{i} H_{\nu}^{ \pm j} W^{\mp}, & \quad \frac{i}{2} g Z^{i 2}\left(p^{H_{\nu}^{i}}-p^{H_{\nu}^{ \pm j}}\right)_{\mu}, \\
\nu^{i} N^{j} H_{\nu}^{k}, & -i \sum_{a=1}^{3} \sum_{b=1}^{3} U_{N}^{i a} U_{N}^{j 3+b} y_{\nu}^{a b} Z^{k 2},
\end{array}
$$

where $U_{L}^{i j}, U_{N}^{i j}$, and $Z^{i j}$ are the charged lepton, neutrino, and $C P$-even neutral Higgs mixing matrices, the bases of the mass matrices being $\{e, \mu, \tau\},\left\{\nu_{e}, \nu_{\mu}, \nu_{\tau}, N_{e}^{c}, N_{\mu}^{c}, N_{\tau}^{c}\right\}$, and $\left\{\phi, \phi_{\nu}\right\}$, respectively. Note that $U_{L}^{i j}$ is a diagonal matrix.

\section{APPENDIX B: LEPTON FLAVOR VIOLATING BRANCHING RATIOS}

In Table $\mathrm{V}$ we have shown the obtained branching ratios for various lepton flavor violating processes corresponding to the four benchmark points. $\operatorname{BR}(\mu \rightarrow e \gamma)$ is projected to be probed experimentally up to $6.0 \times 10^{-14}$ in the near future [66]. As indicated by the numbers, the obtained branching ratios for this process are at least 1 order of magnitude smaller for our benchmark points. The rest of these obtained LFV branching ratios are several orders of magnitude below the present experimental sensitivity in the respective channels. 
TABLE V. Lepton flavor violating branching ratios obtained for our chosen benchmark points.

\begin{tabular}{|c|c|c|c|c|c|c|c|c|}
\hline \multirow{2}{*}{$\begin{array}{l}\text { LFV } \\
\text { Process }\end{array}$} & \multicolumn{2}{|c|}{ BP1 } & \multicolumn{2}{|c|}{ BP2 } & \multicolumn{2}{|c|}{ BP3 } & \multicolumn{2}{|c|}{ BP4 } \\
\hline & Normal & Inverted & Normal & Inverted & Normal & Inverted & Normal & Inverted \\
\hline $\mathrm{BR}(\mu \rightarrow e \gamma)\left(\times 10^{15}\right)$ & 2.97 & 0.79 & 0.56 & 0.21 & 0.81 & 0.31 & 0.99 & 0.38 \\
\hline $\operatorname{BR}(\tau \rightarrow e \gamma)\left(\times 10^{16}\right)$ & 0.38 & 2.05 & 0.10 & 0.56 & 0.15 & 0.80 & 0.18 & 0.98 \\
\hline $\operatorname{BR}(\tau \rightarrow \mu \gamma)\left(\times 10^{15}\right)$ & 2.90 & 3.87 & 0.79 & 1.05 & 1.14 & 1.52 & 1.39 & 1.86 \\
\hline $\mathrm{BR}(\mu \rightarrow$ eee $)\left(\times 10^{17}\right)$ & 1.72 & 0.65 & 0.46 & 0.18 & 0.68 & 0.26 & 0.82 & 0.31 \\
\hline $\operatorname{BR}(\tau \rightarrow$ eеe $)\left(\times 10^{18}\right)$ & 0.51 & 2.73 & 0.14 & 0.73 & 0.20 & 1.07 & 0.24 & 1.30 \\
\hline $\operatorname{BR}(\tau \rightarrow \mu \mu \mu)\left(\times 10^{17}\right)$ & 1.12 & 1.50 & 0.30 & 0.39 & 0.45 & 0.60 & 0.53 & 0.71 \\
\hline
\end{tabular}

[1] G. Aad et al. (ATLAS Collaboration), Phys. Lett. B 716, 1 (2012).

[2] S. Chatrchyan et al. (CMS Collaboration), Phys. Lett. B 716, 30 (2012).

[3] M. C. Gonzalez-Garcia and M. Maltoni, Phys. Rep. 460, 1 (2008).

[4] P. Minkowski, Phys. Lett. 67B, 421 (1977).

[5] R. N. Mohapatra and G. Senjanovic, Phys. Rev. Lett. 44, 912 (1980).

[6] M. Gell-Mann, P. Ramond, and R. Slansky, CERN Report No. Print-80-0576.

[7] T. Yanagida, in Proceedings of the Workshop on the Baryon Number of the Universe and Unified Theories, Tsukuba, Japan, 1979 (National Lab for High Energy Physics, 1979), p. 109.

[8] S. L. Glashow, NATO Adv. Study Inst. Ser. B Phys. 59, 687 (1980).

[9] J. Schechter and J. W. F. Valle, Phys. Rev. D 25, 774 (1982).

[10] J. Schechter and J. W. F. Valle, Phys. Rev. D 22, 2227 (1980).

[11] S. Weinberg, Phys. Rev. Lett. 43, 1566 (1979).

[12] S. Weinberg, Phys. Rev. D 22, 1694 (1980).

[13] W.-Y. Keung and G. Senjanovic, Phys. Rev. Lett. 50, 1427 (1983).

[14] A. Datta, M. Guchait, and A. Pilaftsis, Phys. Rev. D 50, 3195 (1994).

[15] F. M. L. Almeida, Jr., Y. do Amaral Coutinho, J. A. Martins Simoes, and M. A. B. do Vale, Phys. Rev. D 62, 075004 (2000).

[16] O. Panella, M. Cannoni, C. Carimalo, and Y. N. Srivastava, Phys. Rev. D 65, 035005 (2002).

[17] T. Han and B. Zhang, Phys. Rev. Lett. 97, 171804 (2006).

[18] F. del Aguila, J. A. Aguilar-Saavedra, and R. Pittau, J. High Energy Phys. 10 (2007) 047.

[19] K. Huitu, S. Khalil, H. Okada, and S. K. Rai, Phys. Rev. Lett. 101, 181802 (2008).

[20] A. Atre, T. Han, S. Pascoli, and B. Zhang, J. High Energy Phys. 05 (2009) 030.

[21] C.-Y. Chen and P. S. B. Dev, Phys. Rev. D 85, 093018 (2012).
[22] P. S. B. Dev, A. Pilaftsis, and U.-k. Yang, Phys. Rev. Lett. 112, 081801 (2014).

[23] A. Das, P. S. B. Dev, and R. N. Mohapatra, Phys. Rev. D 97, 015018 (2018).

[24] A. Abulencia et al. (CDF Collaboration), Phys. Rev. Lett. 98, 221803 (2007).

[25] S. Chatrchyan et al. (CMS Collaboration), Phys. Lett. B 717, 109 (2012).

[26] CERN Technical Report No. ATLAS-CONF-2012-139, https://atlas.web.cern.ch/Atlas/GROUPS/PHYSICS/ CONFNOTES/ATLAS-CONF-2012-139/.

[27] V. Khachatryan et al. (CMS Collaboration), Phys. Lett. B 748, 144 (2015).

[28] P. S. Bhupal Dev, R. Franceschini, and R. N. Mohapatra, Phys. Rev. D 86, 093010 (2012).

[29] F. F. Deppisch, P. S. Bhupal Dev, and A. Pilaftsis, New J. Phys. 17, 075019 (2015).

[30] M. Magg and C. Wetterich, Phys. Lett. 94B, 61 (1980).

[31] Technical Report No. ATLAS-CONF-2015-044, https:// atlas.web.cern.ch/Atlas/GROUPS/PHYSICS/CONFNOTES/ ATLAS-CONF-2015-044/.

[32] G. C. Branco, P. M. Ferreira, L. Lavoura, M. N. Rebelo, M. Sher, and J. P. Silva, Phys. Rep. 516, 1 (2012).

[33] E. Ma, Phys. Rev. Lett. 86, 2502 (2001).

[34] S. Gabriel and S. Nandi, Phys. Lett. B 655, 141 (2007).

[35] S. M. Davidson and H. E. Logan, Phys. Rev. D 80, 095008 (2009).

[36] S. Gabriel, B. Mukhopadhyaya, S. Nandi, and S. K. Rai, Phys. Lett. B 669, 180 (2008).

[37] N. Haba and K. Tsumura, J. High Energy Phys. 06 (2011) 068.

[38] N. Haba and T. Horita, Phys. Lett. B 705, 98 (2011).

[39] W. Chao and M. J. Ramsey-Musolf, Phys. Rev. D 89, 033007 (2014).

[40] U. Maitra, B. Mukhopadhyaya, S. Nandi, S. K. Rai, and A. Shivaji, Phys. Rev. D 89, 055024 (2014).

[41] S. Chakdar, K. Ghosh, and S. Nandi, Phys. Lett. B 734, 64 (2014).

[42] O. Seto, Phys. Rev. D 92, 073005 (2015).

[43] E. Bertuzzo, Y.F. Perez G., O. Sumensari, and R. Zukanovich Funchal, J. High Energy Phys. 01 (2016) 018. 
[44] C. Guo, S.-Y. Guo, Z.-L. Han, B. Li, and Y. Liao, J. High Energy Phys. 04 (2017) 065.

[45] G. 't Hooft, NATO Sci. Ser. B 59, 135 (1980).

[46] Planck Collaboration, Astron. Astrophys. 594, A13 (2016).

[47] S. Zhou, Phys. Rev. D 84, 038701 (2011).

[48] M. Sher and C. Triola, Phys. Rev. D 83, 117702 (2011).

[49] Ya. B. Zeldovich, I. Yu. Kobzarev, and L. B. Okun, Zh. Eksp. Teor. Fiz. 67, 3 (1974) [Sov. Phys. JETP 40, 1 (1974)].

[50] W.-M. Yao et al., J. Phys. G 33, 1 (2006).

[51] M. C. Gonzalez-Garcia, M. Maltoni, and T. Schwetz, Nucl. Phys. B908, 199 (2016).

[52] P. F. de Salas, D. V. Forero, C. A. Ternes, M. Tortola, and J. W. F. Valle, arXiv:1708.01186.

[53] J. A. Casas and A. Ibarra, Nucl. Phys. B618, 171 (2001).

[54] A. Pilaftsis, Z. Phys. C 55, 275 (1992).

[55] W. Grimus and L. Lavoura, Phys. Lett. B 546, 86 (2002).

[56] D. Aristizabal Sierra and C. E. Yaguna, J. High Energy Phys. 08 (2011) 013.

[57] D. Aristizabal Sierra, Proc. Sci., EPS-HEP2011 (2011) 435 [arXiv:1110.6435].

[58] D. Aristizabal Sierra, J. Phys. Conf. Ser. 375, 042003 (2012).

[59] CERN Technical Report No. ATLAS-CONF-2016089, https://atlas.web.cern.ch/Atlas/GROUPS/PHYSICS/ CONFNOTES/ATLAS-CONF-2016-089/.

[60] A. McCarn (ATLAS CMS Collaborations), in Proceedings, 51st Rencontres de Moriond on Electroweak Interactions and Unified Theories: La Thuile, Italy, 2016, pp. 307-316, http://inspirehep.net/record/1591250/files/1589812_307316.pdf.

[61] H. Ohman (ATLAS CMS Collaborations), in Fourth Annual Large Hadron Collider Physics, Lund, Sweden, 2016, http:// cds.cern.ch/record/2214130/files/ATL-PHYS-PROC-2016137.pdf?subformat=pdfa.

[62] A. G. Akeroyd et al., Eur. Phys. J. C 77, 276 (2017).

[63] G. Aad et al. (ATLAS Collaboration), J. High Energy Phys. 05 (2014) 071.

[64] CMS Collaboration (2017), https://cds.cern.ch/record/ $2297116 ? \ln =$ en.

[65] J. Adam et al. (MEG Collaboration), Phys. Rev. Lett. 110, 201801 (2013).

[66] A. M. Baldini et al., arXiv:1301.7225.
[67] B. Aubert et al. (BABAR Collaboration), Phys. Rev. Lett. 104, 021802 (2010).

[68] T. Aushev et al., arXiv:1002.5012.

[69] K. Hayasaka et al., Phys. Lett. B 687, 139 (2010).

[70] W. H. Bertl et al. (SINDRUM II Collaboration), Eur. Phys. J. C 47, 337 (2006).

[71] L. Bartoszek et al. (Mu2e Collaboration), arXiv:1501 .05241 .

[72] F. Staub, arXiv:0806.0538.

[73] F. Staub, Comput. Phys. Commun. 181, 1077 (2010).

[74] F. Staub, Comput. Phys. Commun. 182, 808 (2011).

[75] F. Staub, Comput. Phys. Commun. 185, 1773 (2014).

[76] F. Staub, Adv. High Energy Phys. 2015, 840780 (2015).

[77] W. Porod, Comput. Phys. Commun. 153, 275 (2003).

[78] W. Porod and F. Staub, Comput. Phys. Commun. 183, 2458 (2012).

[79] H.-J. He, N. Polonsky, and S. Su, Phys. Rev. D 64, 053004 (2001).

[80] J. Alwall, M. Herquet, F. Maltoni, O. Mattelaer, and T. Stelzer, J. High Energy Phys. 06 (2011) 128.

[81] J. Alwall, R. Frederix, S. Frixione, V. Hirschi, F. Maltoni, O. Mattelaer, H. S. Shao, T. Stelzer, P. Torrielli, and M. Zaro, J. High Energy Phys. 07 (2014) 079.

[82] R. D. Ball et al., Nucl. Phys. B867, 244 (2013).

[83] R. D. Ball et al. (NNPDF Collaboration), J. High Energy Phys. 04 (2015) 040.

[84] https://cp3.irmp.ucl.ac.be/projects/madgraph/wiki/FAQGeneral-13.

[85] T. Sjostrand, S. Mrenna, and P. Z. Skands, J. High Energy Phys. 05 (2006) 026.

[86] J. de Favereau, C. Delaere, P. Demin, A. Giammanco, V. LemaÃőtre, A. Mertens, and M. Selvaggi (DELPHES 3 Collaboration), J. High Energy Phys. 02 (2014) 057.

[87] M. Selvaggi, J. Phys. Conf. Ser. 523, 012033 (2014).

[88] A. Mertens, J. Phys. Conf. Ser. 608, 012045 (2015).

[89] M. Cacciari, G. P. Salam, and G. Soyez, Eur. Phys. J. C 72, 1896 (2012).

[90] M. Cacciari, G. P. Salam, and G. Soyez, J. High Energy Phys. 04 (2008) 063.

[91] CERN Technical Report No. ATL-PHYS-PUB-2015022, https://atlas.web.cern.ch/Atlas/GROUPS/PHYSICS/ PUBNOTES/ATL-PHYS-PUB-2015-022/. 